\title{
Use of a Focused Ion Beam for Characterizing SIS Circuits
}

\author{
Robert B. Bass, William W. Clark IV, Jian Z. Zhang, and Arthur W. Lichtenberger
}

\begin{abstract}
We have found the use of a Ga+ based focused ion beam (FIB) system to be very useful in characterizing our superconducting-insulating-superconducting (SIS) fabrication process. This tool enables us to physically carve cross sections in any feature of interest on our wafer which we can then image with an SEM. This process is used to examine and monitor improvements in the coverage of metalization layers over different circuit topography and in the critical "sealing" capabilities of our $\mathrm{SiO}$ insulation layer around the perimeter of the $\mathrm{Nb}$ junction counter electrode. It has also been used to better establish a submerged trilayer deposition process where the base electrode is imbedded in the quartz substrate. We have also improved our characterization of the tunnel barrier critical current density of our $\mathrm{Nb} / \mathrm{Al}$-oxide/ $\mathrm{Nb}$ trilayer material by obtaining more accurate diameter measurements from FIB sectioned junctions.
\end{abstract}

Index Items || characterization, critical current density, cross section, and focused ion beam.

\section{INTRODUCTION}

In our laboratory we use a $\mathrm{Ga}+$ based focused ion beam (FIB) system for the fabrication of SIS junctions and Nb based hot electron bolometers $[1,2]$. We also find the FIB system to be very useful in characterizing our superconductinginsulating-superconducting (SIS) fabrication process. Often the images of a wafer topography obtained with a scanning electron microscope (SEM) are insufficient for a full understanding of thin film coverage or device dimensions.

\section{EXPERIMENTS}

A Ga+ based FEI series 200 [3] FIB system with a $5 \mathrm{~nm}$ beam size is used for this work. The FIB operates much as a scanning electron microscope, imaging secondary electrons ejected from a sample bombarded by gallium ions. Theseions, however, are much more massive than electrons, so that the FIB sputters material from the sample at its point of contact. Typically a pattern is etched in a film by repeatedly removing a thin layer of the entire pattern. The FIB is controlled by CAD

Manuscript received August 2, 2000. This work was supported in part by the National Science Foundation grant No. AST-9987330 and a grant from the National Radio Astronomy Observatory.

W. W. Clark IV, J. Z. Zhang, and A. W. Lichtenberger are with the Department of Electrical Engineering, University of Virginia, Charlottesville VA22903USA( wwc4g@virginia.edu, awl11@virginia.edu, jz4n@virginia.edu).

R. B. Bass was with the University of Virginia, Charlottesville, VA 22903 USA (rbb7y@ hotmail.com). software with user-defined patterns to control such parameters as pixel dwell time, overlap of pixels, total etch time, beam current (eight orders of magnitude) and the actual path the beam travels each time the pattern is written. A change in beam current or a change of strategy in how the beam traverses the same pattern can significantly alter the results of the FIB etch.

To form sections through our SIS circuits, a multiple step process is utilized. After sputtering a thin (5nm) Au layer for improved FIB imaging, the section is cut with a relatively large $150 \mathrm{pA}$ beam current followed by several cuts with beam currents of decreasing size. A final cut at $1 \mathrm{pA}$ is used to polish the face of the section for better subsequent SEM imaging. With our FIB technique, there is approximately 20 minutes of initial FIB set up time, 10 minutes to call up and etch the series of section pattern files and then approximately 3 minutes of set-up time before milling the next section.

The wafers used in this work are all fabricated from Nb/Aloxide/ $\mathrm{Nb}$ trilayer material sputtered in our "tri2" system [4]. The junctions sectioned are either fabricated with our Cr-based [5] or $\mathrm{Nb}$-based [6] trilevel resist process, or a new $\mathrm{Nb}$-based quadlevel resist process. In our trilevel resist process, a single P1 layer of polyimide is used in a $\mathrm{P} 1 / \mathrm{Cr}$ (or $\mathrm{Nb}$ )/imaging-resist etching mask to both define the junctions and as a self-aligned liftoff stencil for the $\mathrm{SiO}$ junction insulation. We have found the

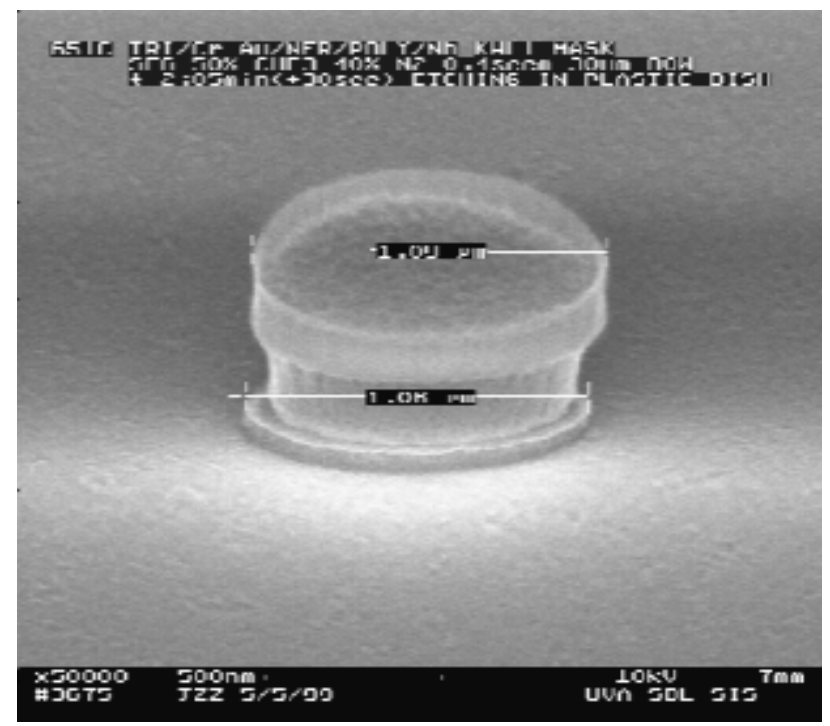

Fig. 1. An etched trilayer/Cr-Au test junction under a quadlevel resist structure before $\mathrm{SiO}$ deposition is shown. It can be seen that the $\mathrm{SF}_{6}+\mathrm{CHF}_{3}$-based $\mathrm{Nb}$ etch is anisotropic. The $\mathrm{Cr}$ - $\mathrm{Au}$ button is clearly seen to be defined by the NFR resist and not the trilevel resist $\mathrm{Nb}$ (which defines the $\mathrm{Nb}$ junction size). 


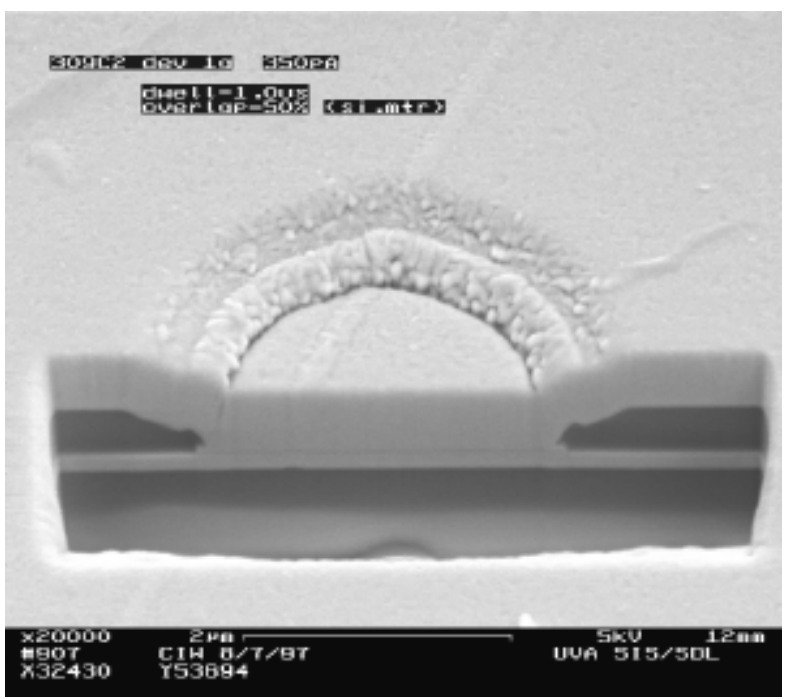

Fig. 2. A cross section of a finished junction clearly showing the extent of the junction counter electrode. The sectioned faces of the $\mathrm{SiO}$ insulation layer and quartz substrate appear dark in the SEM.

anisotropy of this Nb RIE junction counter electrode etch is very dependent on the history and cleanliness of the RIE. This is particularly true for our $\mathrm{Cr}-\mathrm{Au}$ overlayer process [6] where a thin layer of $\mathrm{Cr}-\mathrm{Au}$ is on top of the $\mathrm{Nb}$ counter electrode. It was recently discovered that the presence of NFR resist [7] near the $\mathrm{Nb} / \mathrm{Au}$ feature greatly enhances the anisotropy of our RIE etch. It was also found that the NFR provides a superior mask for our $\mathrm{Cr}$-Au wet etch [8], permitting definition of Cr-Au overlayers for submicrometer sized junctions. After the $\mathrm{NFR}(200 \mathrm{~nm}) /$ polyimide(200nm)/Nb mask is used to define the $\mathrm{Nb} / \mathrm{Cr}-\mathrm{Au}$ counter electrode and an $\mathrm{SiO}$ layer is deposited, the $\mathrm{SiO}$ is lifted off in propylene glycol, which dissolves the polyimide fully but doesn't remove the NFR. The NFR is subsequently removed with a high pressure oxygen plasma etch. An etched trilayer/Au/Cr-Au test junction fabricated

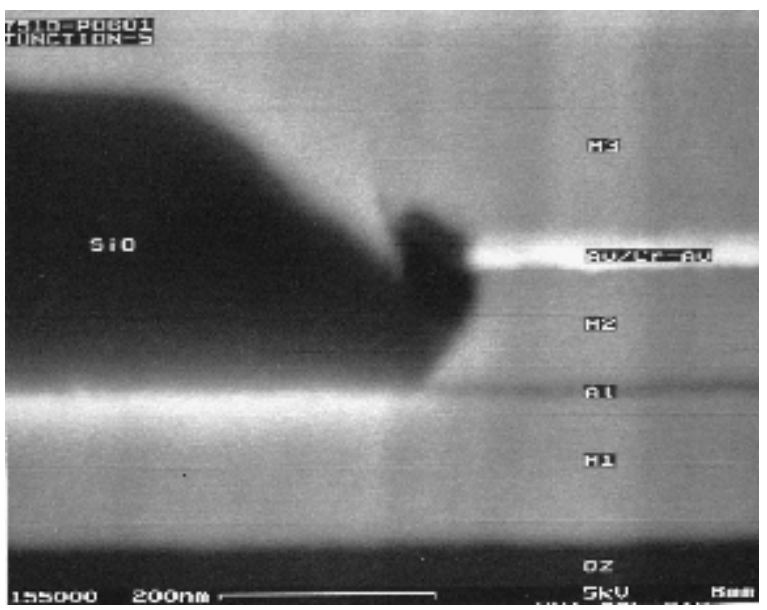

Fig. 3. Cross section of a finished $\mathrm{Cr}-\mathrm{Au}$ overlayer junction for which the isotropic nature of the counter electrode $\mathrm{Nb}$ etch can be seen. This shortcoming was remedied with the use of a quadlevel resist etching mask.

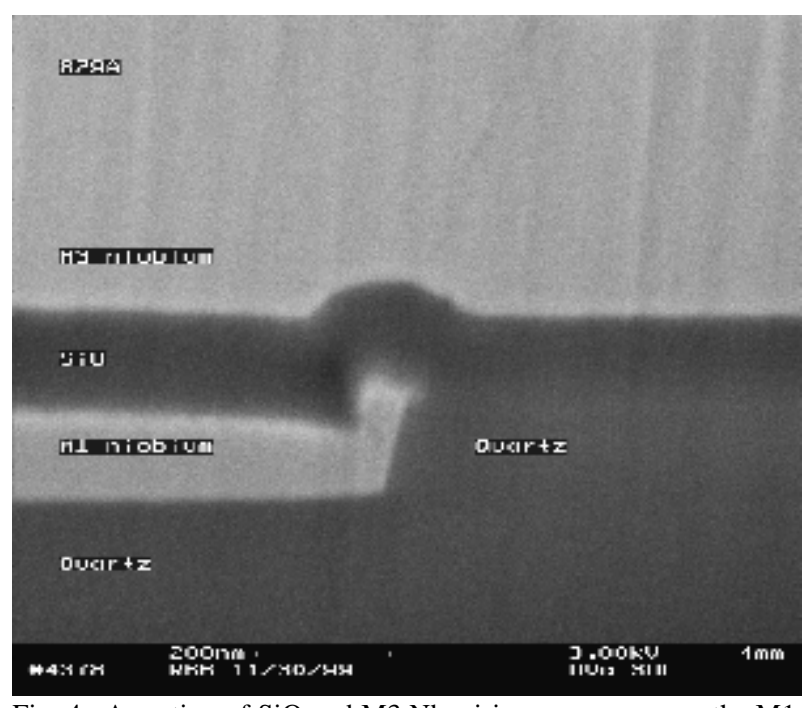

Fig. 4. A section of $\mathrm{SiO}$ and $\mathrm{M} 3 \mathrm{Nb}$ wiring coverage over the $\mathrm{M} 1$ base electrode of a completed wafer where the trilayer was deposited by liftoff in a submerged-trilayer process.

using the quadlevel resist process is shown in Fig. 1. prior to $\mathrm{SiO}$ deposition.

One of our most often used junction diagnostic tests is the sectioning of finished junctions. Fig. 2. shows a typical cross section where the $\mathrm{Nb}$ base and counter electrodes (M1 and M2, respectively), the $\mathrm{SiO}$ insulation (dark) and the $\mathrm{Nb}$ wiring layer (M3) are all clearly defined. It is evident from this SEM that the $\mathrm{SiO}$ insulation is not only successfully sealing the side of the $\mathrm{Nb}$ counter electrode junction button, but is also extending up onto the perimeter of the top surface. Such a conclusion is impossible to make given only an SEM view of the un-sectioned junction. We also use this technique to investigate pinch-off effects in the wiring layer filling of the $\mathrm{SiO}$ vias for submicrometer sized junctions [9]. SEM images of FIB cross sectioned structures are also very helpful in viewing the true profiles of film edges after liftoff or etching. Fig. 3. shows a cross section of a $\mathrm{Cr}-\mathrm{Au}$ overlayer junction for which a polyimide-based trilevel resist etch mask was used. It is evident that the $\mathrm{Nb}$ counter electrode RIE etch was isotropic. This shortcoming was remedied with the use of an NFR based quadlevel resist.

Fig. 4. shows the result of a submerged trilayer process where the trilayer is defined in a trilevel resist liftoff step. The section reveals the $\mathrm{SiO}$ and $\mathrm{M} 3 \mathrm{Nb}$ wiring coverage over the M1 base electrode of a completed wafer. In this process, the trilayer is deposited by liftoff in a submerged process with the intent of making the top of the base electrode level with the quartz surface. Note that the wiring level is planar across the section except for the effect of a small liftoff "wing" at the edge of the base electrode.

As is seen in Fig. 2., it is quite difficult to accurately measure the diameters of un-sectioned $\mathrm{Nb}$ counter electrode buttons in order to calculate the critical current density (Jc) of SIS junctions. In particular, the insulation field covers the base and counter electrode topography in such a way as to distort the 


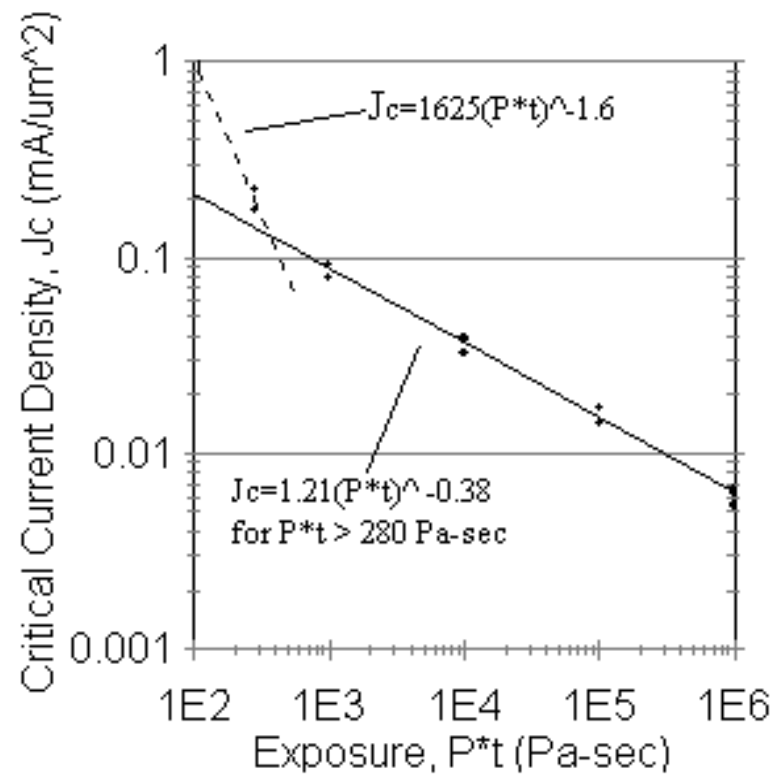

Fig. 5. Critical Current Density, Jc, as a function of oxygen exposure, $\mathrm{E}$ (pressure $\mathrm{x}$ time), for our SIS junctions. A power law fit of $\mathrm{Jc}=1.21(\mathrm{E})^{-0.38}$ for the high $\mathrm{E}$ data give excellent agreement. A tentative fit for the low $\mathrm{E}$ data is also given.

actual location of the perimeter of the junction counter electrode. In contrast, the actual size of the junction electrode can be accurately measured from the SEM of the sectioned junctions.

Fig. 5. shows the dependence of Jc on oxygen dose. The Al layer was oxidized in the trilayer loadlock using pure oxygen for 4-120 minutes at pressures of approximately 1-133Pa. Only junctions with dimensions smaller than the Josephson penetration depth were used such that $I_{c}$ scaled with area. A power law fit to the data, excluding the data at the small exposure of $\mathrm{P} @ / \mathrm{E}=280 \mathrm{~Pa}-\mathrm{sec}$, gives $\mathrm{J}_{\mathrm{c}}=1.21(\mathrm{E})^{-0.38}$. As is seen, the data (excluding the $280 \mathrm{~Pa}-\mathrm{sec}$ points) are in excellent agreement with the fit. This fit is also in excellent agreement with the assembled Jc data by [10] which indicated that Jc is a function of the oxidation pressure-time product in a nearly universal power law dependence. In their work, two regimes are noted: (1) the high dose regime where Jc . A (E) $)^{-0.4}$ and (2) the low dose regime where $\mathrm{Jc}$. $\mathrm{B}(\mathrm{E})^{-1.6}$. While $\mathrm{A}$ and $\mathrm{B}$ vary considerably for the different groups (for the author's data, A $=0.92$ and $\mathrm{B}=1800$ ), much of the assembled data follows the power law exponent of the high dose regime to within $20 \%$. In our work, we interpret the data points at $280 \mathrm{~Pa}-\mathrm{sec}$ as falling in the low does regime and note that the full Jc fit for our high dose regime follows quite closely that of [10]. If we take our one low dose data set, average the measured Jc values and assume a power law dependency of -1.6 [10], a prefactor value of 1530 is obtained, in surprisingly good agreement.

\section{SUMMARY}

We demonstrated the FIB is a very useful tool for characterizing our SIS fabrication process. This tool enables us to physically mill cross sections in any feature of interest on our wafer, which we then image with an SEM. In particular, we used this tool to monitor and improve the anisotropy of our RIE etches, the coverage of our insulation layers and our trilayer liftoff process. We also obtained very accurate measurements of the sizes of finished junctions which enabled us to better characterize our trilayer oxidation process.

\section{REFERENCES}

[1] R.B. Bass and A.W. Lichtenberger, "Focussed Ion Beam Fabrication of Submicron Nb/Al-oxide/Nb Junctions", IEEE Transactions on Applied Superconductivity, 3240-3243, June 1999.

[2] A. Datesman and A.W. Lichtenberger, "Focussed Ion Beam Fabrication of $\mathrm{Nb}$ based Hot Electron Bolometers",IEEE Transactions on Applied Superconductivity, 4237-4240, June 1999.

[3] FEI Company, 7451 NE Evergreen Parkway, Hillisboro, OR 97124

[4] R.S. Amos, P.E. Breyer, H.H Huang, and A.W. Lichtenberger, "Stress and Source Conditions of DC Magnetron Sputtered Nb Films", IEEE Transactions on Applied Superconductivity, 1995

[5] A.W. Lichtenberger, D.M. Lea, C. Li, F.L. Lloyd, R.J. Mattauch, M.J. Feldman, S.-K. Pan, and A.R. Kerr, "Fabrication of Micron Size Artificially Insulated $\mathrm{Nb} / \mathrm{Al}-\mathrm{Al}_{2} \mathrm{O}_{3} / \mathrm{Nb}$ Junctions with a Trilevel Resist Liftoff Process," IEEE Trans. Magn. MAG-27(20), March 1991.

[6] A.R. Kerr, S.-K. Pan, and A.W. Lichtenberger, "A Tunerless SIS Mixer For 200-270 Ghz with Low Output Capacitance", Proceedings of the Ninth International Symposium on Space $\mathrm{THz}$ Technology, 1998.

[7] JSR Microelectronics, 1289 North Mathilda Avenue, Sunnyvale, CA 94089

[8] A.R. Kerr, S.-K. Pan and A.W. Lichtenberger, "A Nb/Al-oxide/Nb Low Noise Integrated Balanced Mixer", Proceedings of the Eleventh International Symposium on Space $\mathrm{THz}$ Technology, May 2000.

[9] R.B. Bass and A.W. Lichtenberger, "Focussed Ion Beam Fabrication of Submicron Nb/Al-oxide/Nb Junctions", IEEE Transactions on Applied Superconductivity, 3240-3243, June 1999.

[10] A.W. Kleinsasser, R.E. Miller, and W.H. Mallison, Dependence of Critical Current Density on Oxygen Exposure in $\mathrm{Nb}-\mathrm{AlOx}-\mathrm{Nb}$ Tunnel Junctions", IEEE Transactions on Applied Superconductivity,26-30, March 1995. 
